\title{
Studies of Electrical Properties of Polyaniline Irradiated by X-rays
}

\author{
Artem Pashchuk, Lilian M. Moya, Sergei A. Paschuk, Hugo R. Schelin, and Carlos M. G. da S. Cruz \\ Federal Center of Technological Education - CEFET/PR, \\ Av. Sete de Setembro, 3165, CEP 80230-901, Curitiba, PR, Brazil
}

Received on 4 August, 2005

\begin{abstract}
Recent development and studies of physical and chemical properties of the conducting polymers have been calling the interest of many scientists. Among this group of materials, the polyaniline (PANI) deserves special attention because of its high chemical and thermal stability under normal conditions, simple process of polymerization and low cost. This polymer is also known due to its strongly pronounced and well studied electrochemical characteristics. In the present work, the variations of the electrical resistance of polyaniline caused by X-rays radiation have been studied. The polymer has been synthesized by electrochemical method in stainless steel and graphite electrodes. The data concerning the electrical resistance of PANI were measured just after the synthesis. This has been performed in the water solution of sulfuric acid within monomer of aniline where the polyaniline has been submitted to the applied voltages in the range from $200 \mathrm{mV}$ to $600 \mathrm{mV}$. Polymeric samples have been irradiated using the $\mathrm{X}$-rays with the energy $50 \mathrm{keV}$ and $100 \mathrm{keV}$. After the irradiation, the electrical measurements have been repeated. The whole experimental procedure was performed several times. The obtained results have clearly shown the potential of polyaniline as a dose sensitive material that could be applied as a radiation sensor.
\end{abstract}

\section{INTRODUCTION}

Recent developments and studies of physical and chemical properties of conducting polymers have been calling the interest of many scientists. Due to their physical and chemical properties, such materials could be used in different areas of science and technology as, for example, detectors and dosimeters of electromagnetic and ionized radiation [1], [2], [3].

Among this group of materials, the polyaniline (PANI) deserves special attention because of its high chemical and thermal stability under normal conditions, simple process of polymerization and low cost. This polymer is also known due to its strongly pronounced and well studied electrochemical characteristics.

The variations of the electrical resistance of polyaniline caused by X-ray radiation have been studied.

\section{METHODOLOGY}

To perform the studies of electric properties of polyaniline and its synthesis, complex electrodes were prepared. They consist of two conducting plates separated by a plastic spacer of variable thickness that was used to control the size of the grown polymer. Because of the low velocity of polymerization the distance between the plates was maintained equal to $0.2 \mathrm{~mm}$. Such construction allows to synthesize a polymer at both electrode elements simultaneously with the subsequent linking of polymeric structures. The scheme of this electrode is represented in Fig. 1. It consists of two parts: contact element and housing. For manufacturing the electrode contact elements stainless steel 304 or graphite MFP-7 was used. In both cases, the materials have been prepared in such a manner, that their working surface had a form of a square with the sides equal to $5 \mathrm{~mm}$. The electrical contact with the measuring equipment was maintained by copper wire connected to graphite or stainless steel electrode elements.

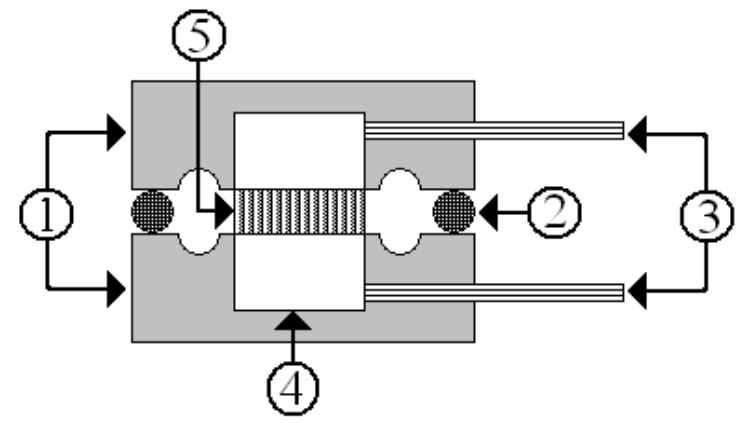

FIG. 1: The scheme of complex electrode: 1 - housing; 2 - plastic spacer; 3 - electrical contact with the measuring equipment; 4 contact elements; 5 - film of polyaniline.

For limiting the area, over which the synthesis of the polymer will occur, the electrode elements have been placed into the housings, which were prepared from resin. Besides this, the housings made it possible to connect electrodes in such a way that the contact elements were located strictly opposite to each other.

The polymer (polyaniline) has been synthesized by electrochemical method where the electrochemical cell with three electrodes, connected to the Potentistat/Galvanostat EG\&G, Model 273A, was used. The synthesis had been performed in an aniline solution $(0.55 \mathrm{~mol} / \mathrm{l})$ within sulfuric acid $(1 \mathrm{~mol} / \mathrm{l})$. The synthesis of polyaniline on graphite electrodes occurred at the voltage range of $0.05 \mathrm{~V}$ to $1.1 \mathrm{~V}$ at the rate of $5 \mathrm{mV} / \mathrm{s}$ during 10 cycles. The synthesis of polyaniline at the stainless electrodes occurred at the voltage range of $0.05 \mathrm{~V}$ to $1.05 \mathrm{~V}$ at rate of $5 \mathrm{mV} / \mathrm{s}$ during 30 cycles. The formation and polymer films had been controlled by comparison of voltammograms obtained separately from each complex electrode element and from both simultaneously.

After the synthesis was concluded, the resistance of the polymer was measured applying a potential of 200, 400 

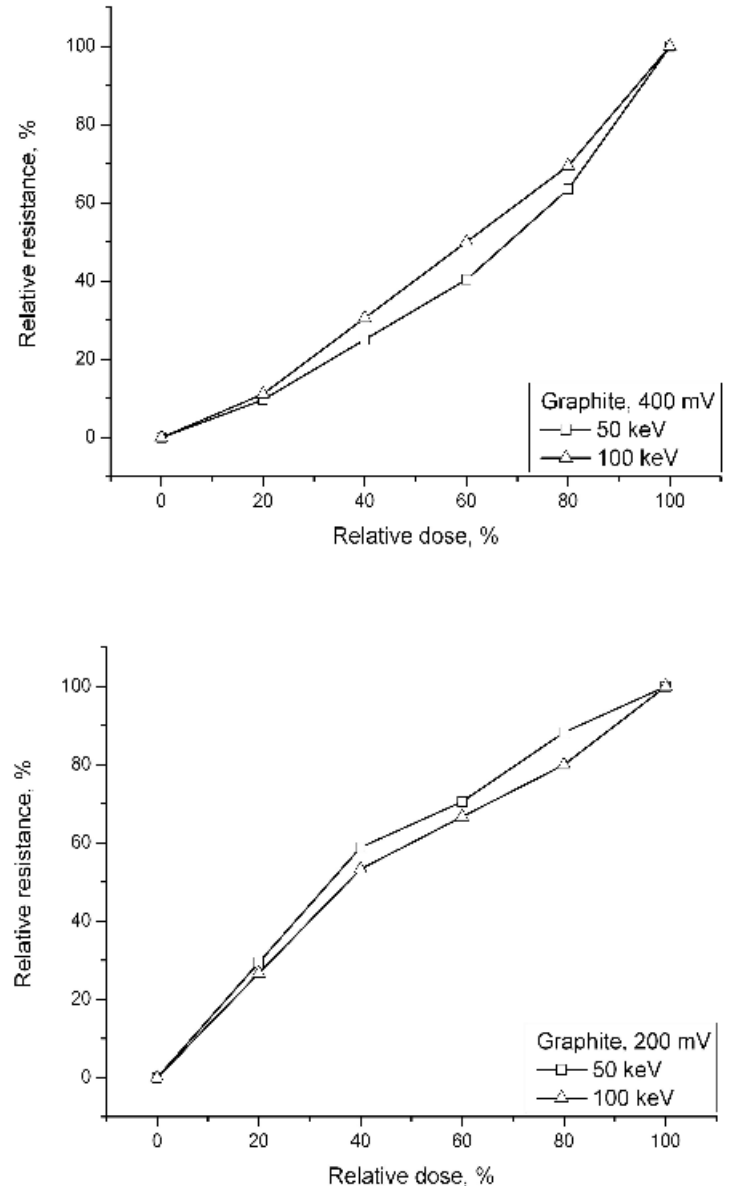

FIG. 2: Experimental curve of electric resistance of polyaniline, synthesized on graphite electrodes, as a function of time of X-ray irradiation with energy $50 \mathrm{keV}$ and $100 \mathrm{keV}$. The polymer polarization voltage had been $600 \mathrm{mV}, 400 \mathrm{mV}$ and $200 \mathrm{mV}$.

and $600 \mathrm{mV}$. At these values of potential there is neither growth nor oxidation of polyaniline. The electrical measurements were performed within aqueous solution of sulfuric acid $(1 \mathrm{~mol} / \mathrm{l})$ within monomer of aniline $(0.55 \mathrm{~mol} / \mathrm{l})$.

The obtained polymer films have then been irradiated by $\mathrm{X}$-ray. At the present work, the X-rays were obtained using a tube with molybdenum anode. The X-ray energy was $50 \mathrm{keV}$ and $100 \mathrm{keV}$ at $5 \mathrm{~mA}$. The polymer samples were placed at $40 \mathrm{~cm}$ from the tube and the time of each exposure was $20 \mathrm{~min}$.

Electrical measurements were repeated after each cycle of irradiation.

\section{RESULTS}

Figures 2 and 3 present the experimental curves refering to the resistance variation of the polymeric films after being irradiated by X-ray with $50 \mathrm{keV}$ and $100 \mathrm{keV}$ energy. These experimental curves were obtained by applying the potential to both extremities of the complex electrode. The values of
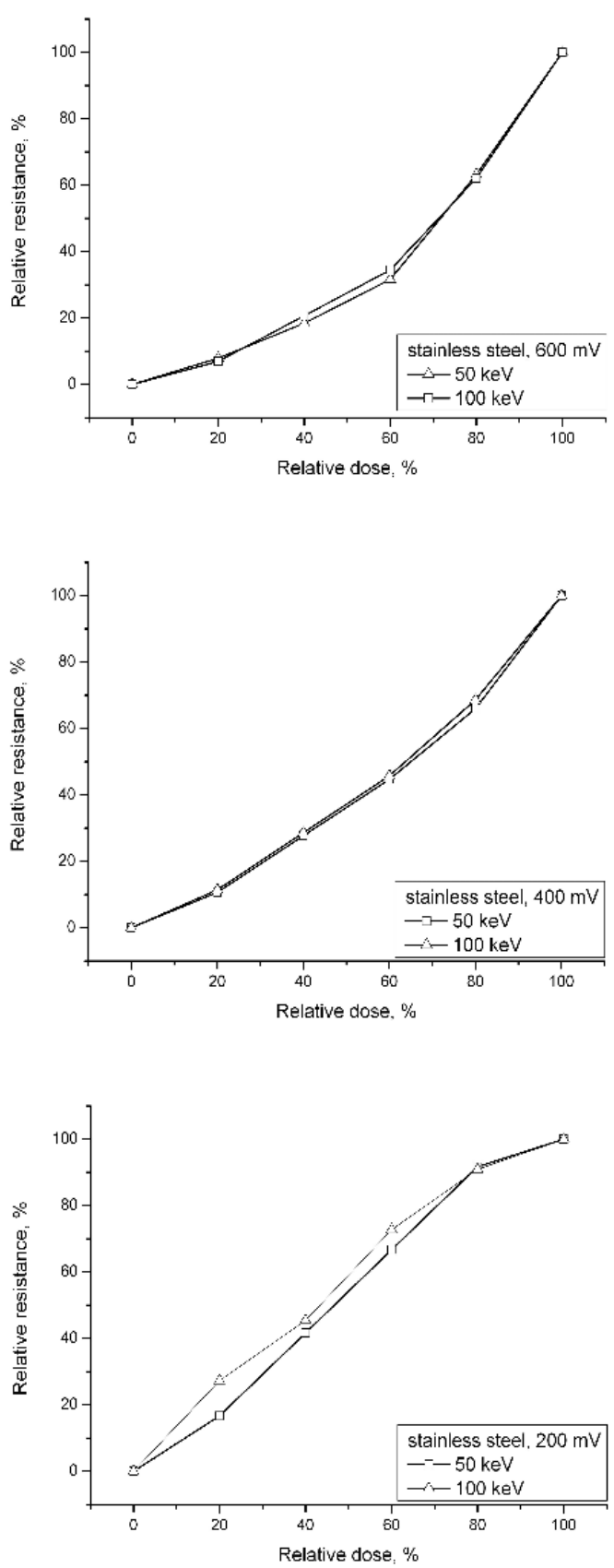

FIG. 3: Experimental curve of electric resistance of polyaniline, synthesized on stainless steel electrodes, as a function of time of $\mathrm{X}$ ray irradiation with energy $50 \mathrm{keV}$ and $100 \mathrm{keV}$. The polymer polarization voltage had been $600 \mathrm{mV}, 400 \mathrm{mV}$ and $200 \mathrm{mV}$.

the potential were equal 200, 400 and $600 \mathrm{mV}$.

Radioactive dose is represented in relative units, because an uncalibrated X-ray tube was used in this work. Resistance is also represented in relative units, because various samples had different initial resistance. So, this mode of representation of results makes it possible to compare changes of the resistance of the different samples. 


\section{DISCUSSION AND CONCLUSIONS}

The obtained results show clearly that the polyaniline is sensitive to the X-ray irradiation. Under the given experimental conditions, it is observed that the polymer is equally sensitive to both energy of X-ray. Also, significant changes of the sensitivity of the polymers films, which were synthesized on different materials, are not observed. However, it is necessary to perform further experiments.

The results of this work unambiguously indicate the possibility of usage of polyaniline films as a basic sensor component in control and measuring equipment for electromagnetic radiation.

The next step will be to compare the results with the chan- ges in the electrochemical properties of polyaniline. Another study of ours show changes in the electrochemical properties of polymer as the function of radiation dose.

Considering the results of other authors [4], we intend to study the sensitivity of polyaniline to electromagnetic radiation that could be changed by introducing impurity of other materials into the structure of a polymeric chain.

\section{Acknowledgments}

The authors are very thankful to the Brazilian agencies $\mathrm{CNPq}, \mathrm{ANP}$ and Fundação Araucária for the financial support.
[1] J. M. G. Laranjeira, H. J. Khoury, W. M. de Azevedo, E. A. de Vasconcelos, and E. F. da Silva Jr., Materials Characterization 5565, 127 (2003).

[2] J. M. G. Laranjeira, H. J. Khoury, W. M. de Azevedo, E. A. de Vasconcelos, and E. F. da Silva Jr. Physica E 17, 666 (2003).
[3] A. P. L. Pacheco, E. S. Araujo, and W. M. de Azevedo, Materials Characterization 50, 245 (2003).

[4] U. A. Sevil, O. Güven, A. Kovacs, and I. Slezsák, Radiation Physics and Chemistry 67, 575 (2003). 